\title{
RELEVANCIA DE LA FILOSOFÍA DE LA EDUCACIÓN EN EL PROCESO DE CONSTRUCCIÓN CURRICULAR DE GRADUACIÓN EN CIENCIAS CONTABLES
}

\section{ARTÍCULO ORIGINAL}

GOMES, Edmar dos Santos ${ }^{1}$, OLIVEIRA, Johnny Jorge de ${ }^{2}$

GOMES, Edmar dos Santos. OLIVEIRA, Johnny Jorge de. Relevancia de la filosofía de la educación en el proceso de construcción curricular de graduación en ciencias contables. Revista Científica Multidisciplinar Núcleo do Conhecimento. Año 07, Ed. 01, Vol. 01, Págs. 58-77. Enero de 2022. ISSN: 2448-0959, Enlace de acceso: https://www.nucleodoconhecimento.com.br/contabilidad/proceso-deconstruccion, DOI: 10.32749/nucleodoconhecimento.com.br/contabilidad/proceso-deconstruccion

\section{RESUMEN}

El artículo presenta una discusión sobre la problemática del proceso de construcción de la estructura curricular de los cursos de graduación en Contabilidad y los principales desafíos en la formación de graduado universitario en Contaduría y busca la respuesta para la pregunta: ¿Cuál es la influencia de este modelo en los currículos de Graduación en Ciencias Contables en el Brasil? Se destaca la injerencia de la filosofía de la educación en esta construcción, cuando los valores y virtudes se colocan en perspectivas indispensables para el ejercicio profesional. El estudio tuvo como objetivo general explorar las suposiciones de la filosofía de la educación y su influencia en la construcción del currículo de formación del profesional contable y específicamente el enfoque de los aspectos históricos de la

\footnotetext{
${ }^{1}$ Licenciada en Ciencias Contables. ORCID: https://orcid.org/0000-0001-7817-2353

${ }^{2}$ Doctorado en Educación. Universidad Católica de Santa Fe, UCSF, Argentina, Magíster en Ciencias Contables y Actuariales. Pontificia Universidad Católica de São Paulo, PUC / SP, Brasil, Especialización en Gestión Pública Universidad Católica Dom Bosco, UCDB, Brasil. Especialización en Docencia de Educación Superior. (Horas: 360h). Universidad Federal de Rio de Janeiro, UFRJ, Brasil. Licenciada en Ciencias Contables. Centro Universitario de Goiás, UNIGOIÁS. ORCID: https://orcid.org/0000-0002-6903-188X
}

RC: 104618

Disponible: https://www.nucleodoconhecimento.com.br/contabilidad/proceso-deconstruccion 
propuesta de reformulación del currículo de las ciencias contables en Brasil y la exploración del pragmatismo existente en estes currículos. Se trata de un estudio de carácter exploratorio-descriptivo, a través de la investigación bibliográfica, aborda los aspectos históricos de la propuesta de reformulación del plan de estudios de graduación en Ciencias Contables, con base en la promulgación de la Ley de Lineamientos y Bases de la Educación Nacional (LDB) n. 9394, de 20 de diciembre de 1996, y actos normativos posteriores. En los resultados se evidenció la interferencia de la Filosofía de la Educación en la construcción de la matriz curricular de Ciencias Contables, cuando se establece la relación entre currículo, conocimientos escolares, su socialización y contextualización, a fin de que puedan ser comprendidos, analizados, criticados y reconstruidos por los alumnos.

Palabras-clave: Filosofía, Ciencias Contables, Currículo, Formación Profesional.

\section{INTRODUCCIÓN}

La creciente expansión de los cursos de Ciencias Contables en el Brasil, en los últimos diez años y la calidad de la enseñanza practicada por Instituciones de Enseñanza Superior (IES), despiertan el interés en discutir el proceso de construcción de la estructura para la formación del profesional de esta área, y como la filosofía de la educación interfiere en esa construcción, cuando se colocan valores y virtudes en perspectiva, indispensables al ejercicio profesional. La cuestión para cual se busca una respuesta en este trabajo es: ¿Cuál es la influencia de este modelo en los currículos de Graduación en Ciencias Contables en el Brasil?

Para un estudio de esa naturaleza, debe ser considerado, inicialmente, el pensamiento de Frigoto (2001), que enfatiza la importancia de tomar conocimiento de la realidad en que se encuentra la educación. Comprender la realidad de la educación, llevando en consideración el aspecto histórico que directamente contribuye con los problemas educacionales, se traduce en la actividad práctica y social de los sujetos históricos concretos. 
Siendo así, el objeto general de este estudio es explorar las suposiciones de filosofía de la educación y su influencia en la construcción del currículo de formación del profesional contable, en un abordaje histórico y conceptual, incluyendo el significado del saber curricular y la constante relación entre teoría y práctica.

Son dos los objetivos específicos de este estudio: en primer plano se procura enfocar los aspectos históricos de la propuesta de reformulación del currículo de graduación de Ciencias Contables, a partir de la promulgación de la Ley de Directrices y Bases de la Educación Nacional (LDB) no 9394, de 20 de diciembre de 1966, y actos normativos posteriores. Se coloca en destaque el papel de la filosofía frente a las nuevas exigencias sociales y los resultados que se espera de la formación profesional contable, pautada en la adquisición de conocimientos, habilidades, competencias y valores que dependen de una enseñanza que establezca un puente entre la teoría y la práctica, ligando los mundos del trabajo y el de la conciencia. Segundo, explorar el pragmatismo existente en los currículos de Ciencias Contables, mediante un análisis de los límites de la educación, cuando observada apenas en la formación para el trabajo. En esta perspectiva de innovación y rescate de autonomía de la universidad, se busca una educación de calidad que incluya también los significados humanos y sociales.

En los aspectos metodológicos del estudio, utilizamos los recursos de la pesquisa bibliográfica sistematizada, del tipo descriptivo y exploratorio. El análisis de carácter exploratorio visa descubrir las semejanzas entre fenómenos estudiados. Las previsiones teóricas no están claras, o son difíciles de encontrar. Según Vergara (2011), en esa situación, se hace un estudio no apenas para conocer el tipo de relación existente, más, sobre todo para determinar la existencia de relación.

Debido a este contexto, la revisión de la literatura sobre el fenómeno estudiado abarca diversos niveles y perspectivas de análisis para la comprensión del tema. Para esto, buscamos el soporte teórico, por medio de levantamiento de la literatura publicada en artículos y textos, libros que tratan del tema central; revistas indexadas, 
otros periódicos, documentos institucionales, como también de disertaciones de máster.

Estudios que abordan esta temática en mucho contribuyen para ampliar el conocimiento sobre el asunto, el cual se reviste de una importancia por traer la comprensión de que la organización curricular de los cursos universitarios se encuentra fundamentada en el paradigma de la educación como proceso permanente, proporcionando al educando las oportunidades de formarse cultural, profesional y técnicamente y, todavía, desarrollarse como persona.

El interés en escoger la temática se justifica una vez que, en las últimas décadas, este asunto fue bastante discutido, tomando proporciones significativas y punto central de debates sobre posibles cambios en el modelo curricular existente, en la perspectiva de que ocurren progresos cuanto a la calidad y productividad de la enseñanza de la Contabilidad. Por tanto, la relevancia de la investigación propuesta advén de la importancia que el profesional contable asume delante del escenario económico y social, por el factor de ser entre otras cosas, el responsable por la mediación del conflicto distributivo existente entre los principales grupos de agentes económicos.

Para Mulatinho (2007) este escenario se debe al proceso de globalización que el sistema capitalista y las economías mundiales están pasando, ligadas al desenvolvimiento acelerado de los diversos medios de comunicación, impactando sobre manera la sociedad y el medio empresarial en toda parte del mundo.

En este contexto, la realización de un estudio que complete el análisis de los contenidos desenvueltos en las universidades, con el objetivo para la formación del profesional contable, exige una necesidad que venga de normas de adaptación, regulación y actuación, pero que sea principalmente pelas amenazas de factores económicos, tales como abertura de mercado, internacionalización de capitales, nuevas modalidades de investimentos, entre otros. 


\section{EL PAPEL DE LA FILOSOFÍA FRENTE A LAS NUEVAS EXIGENCIAS SOCIALES}

La importancia de la Filosofía en el acto de la reflexión permite al hombre ir más allá de la dimensión práctica en la cual está incluido, se transportando para una dimensión transcendental, o sea, de superar situaciones predeterminadas, siendo capaz de construir su destino con libertad La Filosofía transpone el proceso perdido en el inmediatismo de las cosas listas e impide la estagnación del hombre en el mundo. El pensamiento filosófico considera los valores sociales, morales, éticos y políticos como una base que debe nortear las acciones humanas (CHAUÍ, 2003).

Siendo así, el estudio de la Filosofía es esencial en la medida que considera que todo ser humano sea convidado a pensar y actuar a partir de una conducta moral y política que pueda interferir en la sociedad de forma positiva y justa. Con eso, solamente será posible a través del despertar de la consciencia crítica, que buscará descubrir las formas de dominación que se esconde bajo del convencionalismo, de la alienación y de la ideología. En este sentido "[...] la reflexión filosófica y política y su diseminación en los diferentes espacios sociales, posibilita algunas alternativas políticas de resistencia, en que el pensar en su totalidad como un sistema abierto, no sufoque la diversidad" (GENRO, 1999, p. 42).

Para Ferreira (2007) en el contexto actual, marcado por abundancia de conocimientos técnicos y científicos, la Filosofía ejerce importancia crucial. Los valores, expectativas, formas de pensar y actuar son alterados, haciendo surgir en la humanidad posmoderna, personas ansiosas con el suceso inmediato. El ser humano ya no establece relaciones de humanidad para con los otros, viviendo en un mundo marcado por contradicciones y desafíos.

En el entender de Aranha (2006) no existe como educar fuera del mundo. Ningún educador, ninguna institución educacional puede colocarse al margen del mundo. La educación, de cualquier modo, que la entendamos, sufrirá necesariamente el 
impacto de los problemas de la realidad en que ocurre bajo pena de no ser educación.

Es fundamental una re-educación del ser humano, educación esta direccionada para la autonomía, la ética, el respeto a la diversidad cultural y para la búsqueda de la identidad. Uno de los desafíos de esta educación a ser construida es priorizar la cualificación técnica eficiente, capaz de llevar las personas a ocuparen puestos de trabajo cada vez más disputados y desafiantes.

Según Montoya y Pacheco (2003, p. 103)

las actividades profesionales emergentes en la sociedad del conocimiento exigen personas actualizadas. Estas personas deben tener un perfil interdisciplinar, deben ser capaces de buscar sus propias respuestas, y sean dotadas de un espíritu innovador, con capacidad de ser líder y de trabajar en equipo, para poder adaptarse a las mudanzas y acompañarlas con creatividad y responsabilidad.

Sin embargo, se torna imprescindible que el hombre no pierda su dimensión humana. Si en otras épocas ser filósofo era ser amante de la sabiduría, actualmente, este concepto debe ser ampliado y concretizado.

Es preciso hacer una síntesis entre la teoría y la práctica, no pudiendo separar el pensador del agente transformador y este no podrá ser un mero realizador de tareas determinadas por los "sabios". Es importante una formación que pueda ver el otro como un aliado importante para la construcción del saber, se tornando alguien capaz de analizar, evaluar, argumentar y buscar soluciones.

Nuevamente Montoya y Pacheco (2003, p. 101) destacan que:

el aprender se tornó el foco central en las instituciones educacionales de la sociedad del conocimiento. No se quiere más un alumno pasivo, oyente y repetitivo, mas, si, un alumno capaz de aprender a producir conocimiento proprio y, principalmente, ser capaz de utilizar el conocimiento en situaciones que se presentan a lo largo de su vida.

Es necesario comprender todavía que el ser humano es un ser de razón, más también de emoción, capaz de asumir una nueva postura de vida, de usar su libertad 
con más responsabilidad y capaz de proponerse a construir un mundo mejor, más feliz, justo y ético.

En este sentido la universidad tiene un papel preponderante en la vida de todo y cualquier individuo, pues es en ella que el saber encuentra el espacio necesario para su construcción, auxiliando en el desenvolvimiento de competencias intelectuales y, tan importante cuanto eso, para el ejercicio de la ciudadanía.

Compete a las universidades cuidar por la calidad del trabajo académico que realiza y por la competencia de los profesionales que forma. De este modo, "[...] el sentido esencial de la responsabilidad social de la educación de nivel superior consiste en producir y socializar conocimientos que tengan no solamente merito científico, más también valor social y formativo" (DIAS SOBRINHO, 2005, p. 172).

En este contexto, la institución universitaria se tornó foco de las atenciones, por cuestionamiento de la calidad del conocimiento en ella producida y de los procesos educativos por los cuales es responsable, que sean, la diseminación del conocimiento científico y la formación de profesionales y ciudadanos en diferentes áreas.

\section{CONCEPCIONES DE FILOSOFÍA DE LA EDUCACIÓN}

Considerando la Filosofía una reflexión crítica sobre una determinada realidad, se debe entender por realidad todo aquello que existe, esto es, los acontecimientos como ellos son realmente y no como deberían ser. A sí, la Filosofía de la Educación se debruza sobre la realidad de la Educación en su sentido más amplio, sin embargo, variando de pensador para pensador.

Para Saviani (2007, p. 28)

la tarea de la Filosofía de la Educación es ofrecer a los educadores un método de reflexión que les permitan enfrentar los problemas educacionales, penetrando en su complejidad. Esto presupone que la Filosofía de la Educación sea mucho más que un método para educadores, más, sin duda,

RC: 104618

Disponible: https://www.nucleodoconhecimento.com.br/contabilidad/proceso-deconstruccion 
una luz capaz de mostrar la causa y los efectos, y las soluciones de problemas en la realidad educacional.

John Dewey (considerado el exponente máximo de la escuela progresiva americana), es pragmático cuando se trata de su Filosofía de la Educación, la cual se articula alrededor de una Teoría de la Experiencia, vista como intercambio entre sujeto y naturaleza. Para Magalhães (2006) esta teoría se contextualiza con el aprendizaje significativa, esto es, el educando se relaciona con contenidos que están en su cotidiano. Por tanto, el aprendizaje significativo está ligado de forma directa o indirecta a la vida del estudiante y por eso se torna interesante, práctica y útil, pues sabe el alumno, que lo que aprende será utilizado a cualquier momento de su existencia, o sea, él se ve en aquello que está aprendiendo.

Para Dewey, la Educación es la liberación del ser humano frente a la expansión capitalista, o sea, un instrumento capaz de direccionar racionalmente el individuo en sus actividades en el seno de la sociedad, formando un ciudadano crítico y apto a pensar sobre sí mismo y su contexto, o sea, el Filósofo planeaba una Educación extremamente direccionada para la práctica, más sin ignorar la teoría. De un lado el aprendizaje que puede generar significado e traer reflexiones entorno del medio donde vive y aprende el alumno y de otro el pragmatismo, que solamente concibe una acción, o teoría, cuando ésta se efectiva para un fin practico en la vida de quien la recibe $o$ aplica.

Sobre este punto de vista, se destaca la fuerte esencia deweyana que caracteriza el aprendizaje significativo como útil, concreta y, alrededor de la Enseñanza Superior. O sea, el educando vive lo que aprende y aprende lo que vive. ¿Más, cual es la influencia de este modelo en los currículos de Graduación en Ciencias Contables en el Brasil?

\section{EVOLUCIÓN DEL PENSAMIENTO CONTABLE}

Xavier Filho (2008) afirma que la evolución del conocimiento contable encuentra en cuestionamiento cuanto, a su clasificación en cuanto ciencia, pues muchos 
estudiosos la clasifican como una práctica, no siendo unánime el entendimiento de la característica intrínseca como ciencia. El origen del pensamiento contable y su desenvolvimiento primitivo pueden promover un adecuado esqueleto teórico y práctico, que nos permite clasificarla como ciencia, yendo más lejos, ligando la contabilidad a su función social, función que catalizó su surgimiento. Para algunos, no pasa de un arte, para otros, una técnica de registro, mismo sabiendo que la contabilidad atiende a los requisitorios con plenitud, para tornarse una ciencia, por su conjunto de principios, normas y procedimientos propios, cuyo objetivo consiste en conocer la situación patrimonial de las entidades y sus mutaciones.

El Consejo Federal de Contabilidad, en la Resolución CFC n. 774, de 16 de diciembre de 1994, art. 1.1, establece que la contabilidad es

[...] una ciencia con objeto propio - el Patrimonio de las Entidades - y consiste en conocimientos obtenidos por metodología racional, con las condiciones de generalidad, seguridad y busca de las causas, en nivel de calidad semejante a las demás ciencias sociales.

Para ludícibus; Martins e Gelbcke (2003) la contabilidad es presentada como ciencia social. De hecho, ella no podría recibir otra clasificación, como una ciencia exacta, por ejemplo, ya que su objeto de estudio, el patrimonio y sus variaciones, es resultado de acciones humanas. Objetivamente, la Contabilidad comprende un sistema de información y evaluación destinado a fornecer a sus usuarios con demostraciones y análisis de naturaleza económica, financiera, física y de productividad, con relación a la entidad objeto de contabilización.

Hoog (2013) afirma que la ciencia contable siempre fue favorecida por una rica fuente de informaciones, la escrituración, oriunda de los fenómenos de movimiento de la riqueza, que primariamente exigió una preocupación constante de registros, facilitando, de esta forma, la memoria de los actos y eventos, posibilitando el surgimiento de los estudios avanzados de la ciencia de la contabilidad.

Las soluciones superiores que viene del raciocinio contable pasaron a ofrecer medios para se produzcan modelos de gestión de la riqueza. $\mathrm{Y}$, de esta forma, el 
contador dejó de ser apenas un escribano para transformarse en un consejero de las cedulas sociales, orientando y opinando sobre las estrategias de financiamiento, aplicaciones y de los destinos de los emprendimientos.

Para Hoog (2013, p. 12)

la filosofía contable tiene como misión explicar la esencia del saber científico, estudiar y clasificar los fenómenos, en la medida en que se alejan los sofismas; de modo general, la investigación filosófica de la contabilidad valida el objetivo de su conocimiento.

Sá (1997) destaca que varias fueron las escuelas de pensamiento contable, pero no todas trajeron avanzos en la esencia del estudio de la Ciencia Contable, alterando, apenas, la forma de presentación de las corrientes desarrolladas. La literatura describe la evolución del pensamiento contable pasando por varias escuelas: Pseudo-personalismo, Contismo, Materialismo Substancial, Personalismo, Controlismo, Aziendalismo, Reditualismo.

\section{PATRIMONIALISMO, UNIVERSALISMO Y NEO-PATRIMONIALISMO}

Al analizar la filosofía de la contabilidad, considerando sus principales aspectos de evolución, y su importancia en el desenvolvimiento del pensar, Hoog (2013) muestra su pretensión en contribuir con la formación de una filosofía contable contemporánea, admitiendo en su plenitud social y ambiental. Se prestigia la importancia del desenvolvimiento de las teorías y corrientes de pensamientos contables. Particularmente el patrimonialismo de Vincenzo Masi y su expansión que es conocida como el neo-patrimonialismo de Lopes de Sá (1997) y en términos contemporáneos: la teoría pura de la contabilidad, actualmente, lanzan sólidas raíces en el fértil terreno de la filosofía contable.

El neo-patrimonialismo es una doctrina que visa un estudio filosófico de los fenómenos patrimoniales y sus modificaciones, debido a la influencia de los ambientes internos y externos, los cuales operan sobre la riqueza de las empresas. En esa doctrina, la organización lógica de las relaciones que son responsables por 
los orígenes de los acontecimientos está clasificadas en tres grandes grupos de naturaleza: esencial, dimensional y ambiental (SÁ, 1997). El grupo de naturaleza esencial del fenómeno se refiere a las relaciones íntimas de necesidad, finalidad, medios y al aspecto funcional de la riqueza. El dimensional focaliza los aspectos de causa, efecto, tiempo, espacio, calidad y cantidad. En cuanto que, el ambiental se relaciona con la naturaleza administrativa, psíquica, del personal, del social, económica, ecológica, cultural, política, legal, etc. Estas relaciones influencian en la vida del patrimonio.

Delante de la evolución del pensamiento contable, se puede vislumbrar que la filosofía es parte del contexto social que el contador vivencia diariamente, y es lo que genera y agrega valor a una prestación de servicio diferenciada. La filosofía de la contabilidad es imprescindible y altamente relevante para una reflexión rigorosa de seguridad de nuestro conocimiento (HOOG, 2013, p. 26).

\section{EL PRAGMATISMO EN LOS CURRÍCULOS DE CIENCIAS CONTABLES}

La sociedad requiere profesionales de contabilidad capaces de responder los matices de un ambiente de negocios en constante transformación, no solamente para atender las empresas, en lo que se refiere a sus demandas específicas de registros fiscales y acciones de planeamiento y control, pero que también sean capaces de contribuir para el desenvolvimiento del conocimiento científico.

En este contexto, el currículo de graduación visa la formación de profesionales capaces de identificar, criticar, mejorar y aplicar los conocimientos de las ciencias contables, como contraposición a una formación que se preocupa casilque exclusivamente, en preparar el profesional de ciencias contables para apenas viabilizar a los agentes económicos el pleno cumplimiento de su responsabilidad de prestar cuentas de la gestión delante de la sociedad.

ludícibus y Marion (2005, p. 55) destacan

[...] es frecuentemente cometido el error, principalmente en la América Latina, de querer que los cursos formales formen el "profesional pronto y embalado",

RC: 104618

Disponible: https://www.nucleodoconhecimento.com.br/contabilidad/proceso-deconstruccion 
cuando esto, no solamente no es posible, como tal vez, no sea ni mismo deseable. El curso universitario todavía debe ser entendido, básicamente, como un curso de formación que va propiciar las condiciones infraestructurales para que, con experiencia y supervisión posterior, el emergente de tales cursos se torne un día un verdadero profesional.

El curso de Ciencias Contables presenta una preocupación con el dinamismo y las rápidas alteraciones ocurridas en el mundo globalizado. Por consiguiente, busca adaptarse a las reivindicaciones del mercado y buscar proporcionar la calidad de los alumnos de ese curso. El desafío es ampliar el trabajo envolviendo los educadores, en un proceso de integración de las disciplinas del currículo entre sí, con la realidad externa, de modo a superar la fragmentación de la enseñanza.

La definición de currículo emerge del término original proveniente del latín "curriculum" que significa trayecto o camino, sugerir una trayectoria a ser recorrida en la intención de llegar a un destino.

Aunque se entienda, a partir de este concepto, que el currículo es comprendido más allá de la grade de disciplinas que componen un curso, este trabajo parte de una perspectiva conceptual de que el currículo es una construcción social de la escuela, un instrumento por la cual ella delimita un espacio donde van ocurrir las experiencias de enseñanza y aprendizaje, con vistas a un proceso de formación, en un determinado nivel de educación escolar, durante cierto periodo de tiempo (MAIA, 2014).

En sentido amplio, el currículo abarca todas las experiencias escolares y correspondiente a uno de los factores que puede influenciar en la calidad de la enseñanza y en un concepto más amplio de un "[...] conjunto de datos y conocimientos seleccionados de los bienes culturales disponibles, transformados en saber escolar [...] por un proceso que busca adecuar el saber instrumental necesario en respecto a las exigencias económicas, políticas y sociales" (SILVA, 1989, p. 69).

Para Dutra (2003) visto de ese modo, el currículo surge como proceso guiador de la forma de actuación de las instituciones de enseñanza superior, de acuerdo con las 
mudanzas y la restructuración de la sociedad en la cual está inserido. Por tanto, al pensar que el currículo es como un proceso, se defiende la necesidad de su constante reflexión y discusión, para entenderse como una estructura flexible y en permanente (re) construcción.

Para la construcción de un currículo que obedezca la legislación vigente sin se dejar aprisionar por la rigidez por ella expresa, y al mismo tiempo represente la cultura generada por los contextos en que el proceso ocurre, se hace necesario embazarlo en una concepción que permita una flexibilidad para su constante evaluación y reconstrucción. Esto se configura una organización curricular que desenvuelva un lenguaje expresa en delinear, que demuestre el movimiento mantenido a través de la pesquisa y de la extensión, con elementos dinámicos, que se transforman a partir de procesos evaluativos acompañando la dinámica de los contextos sociales.

En 16 de diciembre de 2004, la Resolución o 10 del Consejo Nacional de Educación instituyó las Directrices Curriculares Nacionales para el Curso de Graduación en Ciencias Contables, licenciado, determinando que cada IES debería establecer además de la organización curricular, descrita por medio de proyecto pedagógico, otros aspectos como fértil de los egresados en termos de competencias y habilidades, componentes curriculares, sistemas de evaluación, aprendiz curricular controlado, actividades complementares, el trabajo de conclusión de curso y régimen académico de oferta.

El artículo $2^{\circ}$ de la referida Resolución revela la preocupación en atribuir autonomía y flexibilidad a la enseñanza, y presenta la propuesta de alteración de los currículos de los cursos de graduación en Ciencias Contables en el Brasil. Las IES deberán establecer la organización curricular por medio de Proyectos Pedagógicos, describiendo los siguientes aspectos: a) I - perfil profesional esperando para el formando, en términos de competencias y habilidades; b) II - componentes curriculares integrantes; c) III - sistema de evaluación del estudiante y del curso: d) IV - aprendiz curricular controlado; e) V - actividades complementares: f) VI monografía, proyecto de iniciación científica o proyecto de actividad - como Trabajo 
de Conclusión de Curso (TCC) - componente opcional de la institución: g) VII régimen académico de oferta; h) VIII - otros aspectos que tornen consistente el referido proyecto .

Una análisis breve del contenido de este artículo $2^{\circ}$ nos permite afirmar una analogía con la pedagogía de Dewey que tiene como premisa el permanente contacto entre la teoría y la práctica (praxis); haciendo con que el alumno se torne o momento central del aprendizaje aproximándolo de las pesquisas experimentales, a las cuales la educación debe recorrer para definir correctamente sus propios problemas, además de la construcción de una filosofía de la educación que asuma un papel preponderante en el campo político e social, tenida como eje central en el desenvolvimiento democrático de la sociedad y la formación de un ciudadano dotado de una mentalidad moderna, científica y abierta a la colaboración.

Los principios de las Directrices Curriculares Nacionales aseguran a las IES amplia libertad en la composición de la carga horaria a ser cumplida para la integración de los currículos. Son establecidas las competencias generales, las competencias y habilidades específicas y los contenidos curriculares que contemplen las áreas indicadas para cada curso, incluyendo allí la Práctica Curricular y Actividades Complementares, presentes en el eje de Formación Teórico-Práctico.

Las IES deben contemplar en su Proyecto Político Pedagógico (PPP) el desenvolvimiento de competencias y habilidades de los alumnos, lo que viene al encuentro con el que propone la LDB/96, en el Título I - De la Educación, § 2º, cuando consta que "la educación escolar deberá vincularse al mundo del trabajo y a la práctica social." Esto indica las dimensiones de la vida o contexto valorizados por la LDB: el trabajo y la ciudadanía activa para el ejercicio consciente de los derechos y deberes, en especial la educación en el proceso de construcción social.

Estas ideas indican que la adquisición de conocimientos, habilidades, competencias y valores dependen de una enseñanza que establezca el puente entre la teoría y la práctica, ligando los mundos del trabajo y de la ciencia. 
Guimarães (2009, p. 5316) afirma que la configuración del PPP constituyese un proceso democrático de decisiones que se preocupa en instaurar una forma de organización del trabajo pedagógico que supere los conflictos, buscando eliminar las relaciones competitiva, corporativas y autoritarias e, por su vez, rompiendo con la rutina del mundo impersonal y racionalizado de la burocracia que dificulta las relaciones en el interior de las IES y los efectos fragmentarios de la división del trabajo que refuerza las diferencias y jerarquiza los poderes de decisión .

A sin, el PPP es una acción intencional, con un sentido explicito, con un compromiso defendido colectivamente. Por eso, todo PPP es también, un proyecto político por estar íntimamente articulado al compromiso sociopolítico con los intereses reales y colectivos de la populación mayoritaria. Es político no sentido de compromiso con la formación del ciudadano para un tipo de sociedad (VEIGA, 1995). En la dimensión pedagógica reside la posibilidad de efectividad de la intencionalidad de la universidad, que es la formación del ciudadano participativo, responsable, comprometido, crítico y creativo. Pedagógico también en el sentido de definir las acciones educativas y las características necesarias a las escuelas que cumplan sus propósitos y su intencionalidad.

En esta dirección, la citada Resolución, al describir las Directrices Curriculares Nacionales del Curso Graduación en Ciencias Contables, retrata en el artículo $3^{\circ}$ que el curso debe desear condiciones para que el futuro contabilista sea capacitado a: I - comprender las cuestiones científicas, técnicas, sociales, económicas y financieras, en ámbito nacional e internacional y en los diferentes modelos de organización; II - presentar pleno dominio de las responsabilidades funcionales envolviendo apuraciones, auditorias, pericias, arbitrajes, nociones de actividades actuarías y de cuantificación de informaciones financieras, patrimoniales y gubernamentales, con la plena utilización de innovaciones tecnológicas; III - revelar capacidad crítica-analítica evaluación, cuando a las implicaciones organizacionales con el llegada de la tecnología de la información. 
Teniendo en vista esta formación del profesional del área Contable, y para que un alumno, futuro profesional, salga de la IES con esa amplia visión, de dentro de otras necesidades, se hace necesario existencia de un cuerpo docente comprometido con la enseñanza Contable, con una percepción clara de la realidad y de los fenómenos sociales, políticas, económicas y jurídicos, presentando no solo conocimientos técnicos en Contabilidad y áreas a fines, además también filosóficos, pedagógicos, didácticos y aptitudes sociales entre otros modo a ser capaz de integrar enseñanza, pesquisa y extensión (NOSSA, 1999).

Capacchi et al. (2007), argumentan que no se puede tener un mismo currículo de Ciencias Contables en regiones distintas del país, considerando que el currículo debe reflejar la necesidad del profesional del mercado regional, apuntan que los currículos, por ellos analizados, poseen un volumen muy grande y disciplinas de otras áreas, en detrimento de las disciplinas de áreas de Contabilidad. Los autores cuestionan si los profesionales no están recibiendo formación mucho generalista; que, en realidad, saben un poco de todo y nada con profundidad frente al profesionalismo esperado pelos empresarios.

Otros autores, sin embargo, defienden la multiplicidad de disciplinas de otras áreas en los cursos de Ciencias Contables. A sin, en lugar de la gran especialización del currículo en Contabilidad, se debe buscar la formación de un profesional dinámico, capaz de liderar con la complexidad del mundo actual (GUIMARÃES, 2009).

Leal; Oliveira y Miranda (2010) corroboran la propuesta de la adquisición de conocimiento no solo contable, más también de áreas a fines, pues la relación de la Contabilidad con otras áreas puede contribuir para ampliar los conocimientos del profesional, reforzando habilidades interdisciplinares.

Integrar la composición curricular y el perfil del profesional que el curso desea formar es una cuestión de aliñamiento estratégico para el suceso del curso de graduación. Carvalho y Nakagawa (2005), sin embargo, critican que las IES no consideran las necesidades del mercado cuando de la definición del perfil de profesional a ser 
formado y avisan que esa situación es agravada por ellas no conseguir formar el profesional de acuerdo con la definición planeada.

Marion; Garcia y Cordeiro (2008) destacan un agravamiento del curso de Ciencias Contables es que las disciplinas son repetitivas, a veces sin seguir un proceso lógico secuencial, generando como consecuencia una falta de aptitud por parte de los egresados en ejercer en la práctica su profesión. Atribuye todavía la existencia de muchas disciplinas en la grade curricular que poco contribuye en la formación del profesional contador. Los autores también destacan las limitaciones y dificultades de los alumnos cuanto, al aprendizaje de la Contabilidad Aplicada, en vista de la excesiva concentración de disciplinas de naturaleza técnica, toman la visión del profesional estricto a la Escribano Contable.

En relación a los currículos, los autores arriba citados colocan que el escenario educacional es delicado y apuntan como principales factores la falta de adecuación curricular y acompañada por la falta de un programa definido para la práctica contable está falta de preparo del cuerpo docente, deficiente en la metodología de enseñanza de la Contabilidad introductoria y proliferación de las instituciones de enseñanza y órganos de clase.

\section{CONCLUSIÓN}

En la discusión sobre la problemática del proceso de construcción de la estructura curricular de cursos de graduación y los principales desafíos en la formación del licenciado en Ciencias Contables, se busca destacar la interferencia de la filosofía de la educación en esa construcción, cuando se coloca los valores y virtudes en perspectivas indispensables al ejercicio profesional.

El desenvolvimiento de los objetivos nos lleva a comprender que la estructura curricular de los cursos de Contabilidad es elemento fundamental que delinea las referencias para la construcción de prácticas pedagógicas capaces de producir significados y posibilitar la constitución de identidades sociales y culturales. 
El currículo entendido en su función social proporciona una interacción entre la escuela y la sociedad reproduciendo sus itinerarios históricos o construyendo un espacio de conflictos y compromisos dentro de las contradicciones de estas relaciones. En esta perspectiva, Dewey hizo florecer la idea de educación como proceso social, el instrumento primordial y más eficaz para la construcción social. Este entendimiento de educación y de universidad es guiado por formulación teórica de la cual origina todo un sistema de valores que influencia no solamente el proceso de construcción del conocimiento, más también la manera de ser, de hacer y de vivir/convivir, de hecho, que impone un proceso decisorio colectivo, descentralizando y participando.

De esta forma, el currículo no es apenas un conjunto de las disciplinas que el alumno deba transitar, o sea, el plano de estudios para obtener un diploma, más sin, algo bien más amplio, o sea, un intercambio entre la cultura y la sociedad exterior a la escuela y la educación, entre el conocimiento y la cultura heredados y el aprendizaje de los alumnos y la teoría.

En este sentido y por lo tanto se encontró una respuesta para la pregunta problema, a partir de que se evidenció la interferencia de la Filosofía de la Educación en la construcción de la matriz curricular de Ciencias Contables, cuando se establece la relación entre currículo, conocimientos escolares, su socialización y contextualización, a fin de que puedan ser comprendidos, analizados, criticados y reconstruidos por los alumnos. Es de este modo que serán sujetos activos, capaces de adquirir habilidades necesarias para establecer mudanzas en sus espacios sociales. Por otro lado, eso implica considerar la importancia de la selección de los conocimientos que compondrán la matriz curricular, pues es imprescindible que sean significativos y relevantes para promover la mudanza necesaria y la ampliación del universo cultural del alumno.

Las publicaciones analizadas a respecto de la estructura curricular de los Cursos de Ciencias Contables reportan al entendimiento de que la enseñanza de esta área viene asumiendo característica técnica más objetivando a la práctica de "cómo 
hacer" de que propiamente el desenvolvimiento del espíritu crítico, asociados a los contenidos abordados en disciplinas como filosofía, psicología y ética. Existe un consenso entre los autores de que la academia tiene formados profesionales menos preocupados con las relaciones humanas, pero más capacitados con el ambiente corporativo y la competitividad de los grandes centros empresariales.

En esta perspectiva, se observa la necesidad de repensar el papel de la universidad, delante de su espíritu de innovación y rescate de autonomía, en la búsqueda de una educación de calidad, la cual abarque también los significados humanos y sociales. Este entendimiento corrobora con la idea de que la universidad debe vigilar por la calidad del trabajo académico que realiza y por la competencia de los profesionales que forma. De modo general, debe priorizar el desenvolvimiento de competencias, habilidades, valores y conocimientos en función de los nuevos saberes que se producen y que exigen un nuevo perfil profesional. De esta forma, entendemos un delineamiento al pensamiento de Dewey de que la escuela no podría permanecer ajena a esa profunda transformación social, más sin, se unir al "progreso social" objetivando mudar a su estructura.

A la luz del pensamiento de Dewey y considerando la realidad de la Ciencia Contable en el Brasil y las normas que regulan la actividad profesional, la Universidad debería ser, entre otras, una "sociedad embrionaria", a través de una relación más estrecha con la realidad social del trabajo, uniendo actividades escolares con las productivas introduciendo en el ámbito escolar motivaciones concretas para el aprendizaje de las varias materias, a través de la concientización de su utilidad en el cotidiano.

\section{REFERENCIAS}

ARANHA, Maria Lúcia de Arruda. História da Educação e da Pedagogia: geral e do Brasil. 3. ed. São Paulo: Moderna, 2006. p. 140. 
BRASIL. MINISTÉRIO DA EDUCAÇÃO E CULTURA. RESOLUÇÃO № 10/2004. CÂMARA DE EDUCAÇÃO SUPERIOR - CONSELHO NACIONAL DE EDUCAÇÃO. DIÁRIO OFICIAL DA UNIÃO. BRASÍLIA-DF. Disponible en: http://www.mec.gov.br. accedido en: 10/07/2021.

BRASIL. MINISTÉRIO DA EDUCAÇÃO E CULTURA. LEI DE DIRETRIZES E BASES DA EDUCAÇÃO-LEI №. 9.394. ESTABELECE AS DIRETRIZES E BASES DA EDUCAÇÃO NACIONAL. DIÁRIO OFICIAL DA UNIÃO. BRASÍLIA-DF, 20 DIC 1996.

CAPACCHI, Maristela; MORETTO, Cleide Fátima.; VANCIN, Valmor; PADILHA, Fabio Antônio Rezende (2007). A prática do ensino contábil no Estado do Rio Grande do Sul: uma análise da grade curricular frente às exigências legais e necessidades acadêmicas. In: CONGRESSO ANPCONT, 1.; 2007, Gramado. Anais. Gramado: ANPCONT.

CHAUÍ, Marilena. Convite à Filosofia. 13. ed. São Paulo: Afiliada, 2003.

CARVALHO, Antonio Manuel Rezende; NAKAGAWA, Masayuki. Uma proposta de mudança na formação acadêmica do profissional de custos. Anais Do Congresso Brasileiro De Custos - ABC, 2005. Disponible en: https://anaiscbc.emnuvens.com.br/anais/article/view/1909. Accedido en: 06/08/2021.

DIAS SOBRINHO, José. Dilemas da educação superior no mundo globalizado: sociedade do conhecimento ou economia do conhecimento? São Paulo: Casa do Psicólogo, 2005, p. 172.

DUTRA, Onei Tadeu. Proposta de uma matriz curricular para o curso de Ciências Contábeis na Grande Florianópolis. 2003. Dissertação (Mestrado) Universidade de São Paulo, São Paulo, 2003.

FERREIRA, Márie dos Santos. A natureza filosófica da ética segundo Lima Vaz. Polymatheia, Fortaleza, v. 3, n. 4, p.237-250, 2007. 
FRIGOTO, Gaudêncio. A produtividade da escola improdutiva. Um (re) exame das relações entre educação e estrutura econômico-social capitalista. 6 . ed. SÃO PAULO: CORTEZ, 2001.

GENRO, Maria Ely. Cidadania e subjetividade emancipatória: ação políticoeducativa na universidade. [Seção do Livro] // Pedagogia Universitária: Conhecimento, ética e política no ensino superior / A. do livro LEITE Denise (org.) Porto Alegre: Ed. Universidade/UFRGS, 1999.

GUIMARÃES, Isac Pimentel et al. Uma análise dos projetos político-pedagógicos dos cursos de Ciências Contábeis das universidades públicas do estado da Bahia. Actas do X Congresso Internacional Galego-Português de Psicopedagogia. Braga: Universidade do Minho, 2009, p. 5316.

HOOG, Wilson Zappa. Filosofia Aplicada à contabilidade. Curitiba: Juruá, 2013.

IUDÍCIBUS, Sérgio de; MARION, José Carlos. Introdução à teoria da contabilidade para o nível de graduação. São Paulo: Atlas, 2009.

IUDíCIBUS, Sérgio de; MARTINS, Eliseu; GELBCKE, Ernesto Rubens. Manual de contabilidade das sociedades por ações: aplicável às demais sociedades. [S.I: s.n.], 2003.

LEAL, Edvalda Araújo.; OLIVEIRA, Cintia Rodrigues Medeiros.; MIRANDA, Gilberto José. Interdisciplinaridade no curso de Ciências Contábeis: os desafios e as possibilidades de aprender e ensinar a partir de uma experiência. IN: CONGRESSO ANPCONT, 4., 2010. ANAIS. Natal: ANPCONT, 2010.

MAGALHÃES, Maria Cecilia Camargo. A formação contínua de professores: sessão reflexiva como espaço de negociação entre professores e pesquisador externo. In: MAGALHÃES, Maria Cecilia Camargo; FIDALGO, Sueli Sales; SHIMOURA, Alzira da Silva. Pesquisa Crítica de Colaboração: um Percurso na Formação Docente. São Paulo: Ductor, 2006, p. 97-113. 
MAIA, José Antônio. O currículo no ensino superior em saúde. In: Batista NA, Batista SH. Docência em saúde: temas e experiências. 2.ed. São Paulo: Senac, 2014.

MARION, José Carlos; GARCIA, Elias; CORDEIRO, Morrom. A Discussão sobre a Metodologia de Ensino Aplicável à Contabilidade. Revista do Conselho de Contabilidade de São Paulo, (2008) 10(1), p.28-33.

MONTOYA, Imgard Krüger; PACHECO, Yara de Macedo. Os desafios da Universidade na sociedade do conhecimento. In: BEHRENS, Marilda Aparecida (Org.). Docência universitária na sociedade do conhecimento. Curitiba, Paraná: Champagnat, 2003. p. 103.

MULATINHO, Caio Eduardo Silva. Educação contábil: um estudo comparativo das grades curriculares e da percepção dos docentes dos cursos de graduação das Universidades Federais da Paraíba, Pernambuco e Rio Grande do Norte, referentes ao Programa Mundial de Estudos em Contabilidade proposto pelo ISAR/UNCTAD/ONU. Dissertação (Mestrado em Ciências Contábeis) - Programa multi-institucional e inter-regional de pós-graduação em Ciências Contábeis, da Universidade de Brasília, Universidade Federal de Pernambuco e da Universidade Federal do Rio Grande do Norte. Recife, 2007, p. 254.

NOSSA, Valcemiro. Formação do corpo docente dos cursos de graduação em Contabilidade no Brasil: Uma análise crítica. Cadernos de Estudos FIPECAFI. São Paulo: FEA/USP, v. 11, n. 21, p. 74-92, may/ago. 1999.

SÁ, Antônio Lopes de. História geral e das doutrinas da contabilidade. São Paulo: Atlas, 1997.

SAVIANI, Dermeval. Educação do senso comum à consciência filosófica. 17. ED. Revista Campinas: Cortez Autores Associados, 2007, p. 28. 
VEIGA, IIma Passos Alencastro. Projeto Político-Pedagógico da Escola: uma construção coletiva. In: (org.). Projeto Político-Pedagógico da Escola: uma construção possível. 24à .ed. Campinas: Papirus, 1995.

VERGARA, Sylvia Constant. Projetos e relatórios de pesquisa em Administração. 13. ed. SÃO PAULO: ATLAS, 2011.

XAVIER FILHO, José Lindenberg Julião. Ciências Contábeis uma ciência social aplicada? Pernambuco: Interfaces de Saberes. 2008, p. 1-12.

Enviado: Octubre, 2021.

Aprobado: Enero, 2022. 